\title{
Patients' Expectations in Lens Extraction Surgery: a Systematic Review
}

\author{
Eirini-Kanella Panagiotopoulou*, Panagiota Ntonti, Eleni Vlachou, Kimon Georgantzoglou, \\ Georgios Labiris
}

\begin{abstract}
It is common knowledge that there are patients who have an uncomplicated cataract surgery with an actual improvement of their visual acuity, but they are dissatisfied with their final visual capacity. It is hypothesized that patients' preoperative expectations play a significant role in their postoperative perceptions. A systematic review of the recent literature regarding preoperative expectations of patients before lens extraction surgery and their postoperative perceptions as regards the visual outcome was performed based on the PubMed, Medline, Google Scholar, American Academy of Ophthalmology, Nature and Springer databases in September 2017 and data from 14 descriptive and 7 comparative studies were included in this narrative review. The objective of this review is the determination of the relationship between preoperative expectations and postoperative perception of visual outcome, as well as the investigation of predictors of patient satisfaction by understanding the factors that determine preoperative patient expectations. A considerable number of studies evaluate patient expectations before cataract surgery and compare them with postoperative patient perceptions. In conclusion, the final patient's postoperative perception could be affected both by the actual outcome of the operation and by patient preoperative expectations. Ocular and systemic comorbidity, unrealistic expectations, preoperative spectacle independence, the cost of surgery, and a previous cataract surgery as well as the level of health literacy and age could influence preoperative expectations and predict more accurately patient satisfaction. Taking these factors into consideration could allow surgeons to control the expectations with an extensive preoperative counseling.
\end{abstract}

\section{KEYWORDS}

satisfaction; cataract; expectations; visual outcomes

\section{AUTHOR AFFILIATIONS}

Department of Ophthalmology, University Hospital of Alexandroupolis, Dragana, Alexandroupolis, Greece

* Ophthalmology Department, University Hospital of Alexandroupolis, 68100 Dragana, Alexandroupolis, Greece;

e-mail: eipanagi@med.duth.gr

Received: 2 July 2018

Accepted: 11 November 2018

Published online: 22 January 2019

Acta Medica (Hradec Králové) 2018; 61(4): 115-124

https://doi.org/10.14712/18059694.2018.129

(c) 2018 The Authors. This is an open-access article distributed under the terms of the Creative Commons Attribution License (http://creativecommons.org/licenses/by/4.0), which permits unrestricted use, distribution, and reproduction in any medium, provided the original author and source are credited. 


\section{INTRODUCTION}

Cataract is formed by cloudy areas of accumulated protein on the crystalline lens of the eye. It is mainly an age-related eye disease that hinders clear vision. According to the World Health Organization, 20 million people are blind worldwide because of untreated cataract, namely $51 \%$ of world blindness arises from cataract (1). Currently, there is not any proven prophylactic medical treatment (2). However, reduction of smoking and exposure to ultraviolent radiation could prevent or delay the development of cataract. Other risk factors are diabetes and high BMI (body mass index). The only efficient treatment is surgical removal of the clouded lens and its replacement with an artificial intraocular lens (IOL). Nevertheless, in many developing countries people have no easy access to eye care. Hence cataract still remains the main cause of blindness (1).

Cataract surgery is the most common procedure performed worldwide (3). Today, the most popular technique of surgical removal of cataract in developed countries is phacoemulsification (2). However, when the relatively new technique of phacoemulsification is not easily available, the opaque lens is removed via extracapsular cataract extraction (ECCE). The great safety and efficacy of cataract surgery, the progress in the techniques of ocular biometry measurements and the existence of accurate IOL power calculation methods increase patients' and surgeons' expectations for a continuous improvement of outcomes (4).

The increasing patient expectations for the visual outcomes after a cataract surgery, intensify not only the need for evaluation of patient satisfaction, but also the need for a more detailed and targeted assessment of patient preoperative expectations and postoperative perceptions for the visual outcome.

There is no doubt that a number of patients, who have an uncomplicated cataract surgery with an actual improvement of their visual acuity (VA), are dissatisfied with their final visual function. Probable causes might be insufficient communication between patients and ophthalmologists, high expectations and demographic characteristics of each patient (5).

Regarding the expectations-satisfaction relationship, if the product/service performance is higher than the expected, patients are highly satisfied. If the performance matches individuals' expectations, they are satisfied. If it is lower than their expectations, individuals are dissatisfied $(6,7)$. In order to assess the success of a cataract surgery, the patient-reported outcome and patient satisfaction could be taken into account because they would demonstrate if postoperative visual outcome achieved preoperative expectations (8). Therefore, medical care professionals should control patients' expectations for the outcome of the received treatment in order to affect their perception about the final treatment quality (6).

Aiming a more comprehensive description of preoperative expectations and self-reported level of difficulty and satisfaction with vision after ocular procedures, a variety of questionnaires have been created. Among them are Visual Function 12-Item Scale (VF-12) (4), VF-14 (7-9), VF-15 (10) and Catquest-9SF (11).

Within this context, primary objective of this study is to review the published literature regarding patients' expectations before a lens extraction surgery and to determine the relationship between preoperative expectations and postoperative perception of visual outcome. A secondary objective of the present study is the investigation of predictors of patient satisfaction by understanding the factors that determine preoperative patient expectations. Our intention is the best possible comprehension of the literature regarding patient expectations for a favorable cataract surgery visual outcome and the assistance of other researchers in further investigation in this domain of knowledge.

\section{MATERIAL AND METHODS}

A systematic search for relevant studies was performed based on the PubMed, Medline, Google Scholar, American Academy of Ophthalmology, Nature and Springer databases using the following search terms: preoperative expectations AND cataract surgery, patients expectations AND multifocal intraocular lenses, preoperative expectations AND cataract surgery AND postoperative outcomes. The search took place in September of 2017. Search filters and language restrictions were not used in this initial search. The results of this search were checked and only articles with a relative to the subject title were selected. Afterwards, the abstracts and full texts of these selected articles were reviewed thoroughly and the following data were extracted and assessed: patient selection, demographic characteristics, questionnaires, preoperative expectations, postoperative perceptions, spectacle independence, comorbidity, unrealistic expectations, cost of surgery, personality-psychology, use of spectacles preoperatively and previous cataract surgery. Both comparative and descriptive studies in adult and adolescent patients were included in this review. Articles not available in English, German or Spanish language were excluded. When the eligible articles were not available in full text, abstracts were used as a source of information.

\section{RESULTS}

\section{STUDIES' DESIGN}

The present review included 14 descriptive $(2,4,8,10-20)$ and 7 comparative $(7,9,21-25)$ studies. Among them, 9 were prospective studies $(2,8-13,19,25)$ and 1 retrospective (16), while there was not any relative statement in the rest of the studies (Table 1). One study (9) compared patients' expectations of a group, which was informed by a film about what they could expect from a cataract surgery and of another

Tab. 1 Studies design.

\begin{tabular}{|l|l|l|l|l|}
\hline Studies & Comparative studies & Descriptive studies & Prospective studies & Retrospective studies \\
\hline References & $7,9,21-25$ & $2,4,8,10-20$ & $2,8-13,19,25$ & 16 \\
\hline No. of studies & 7 & 14 & 9 & 1 \\
\hline
\end{tabular}


group, which was enlightened by video giving information on the anatomy and the pathophysiology of the cataract. Additionally, expectations of patients who had already undergone lens exchange and patients who did not have any previous cataract operation were compared (9). One of the objectives in the study of Nijkamp et al. (7) was the comparison of preoperative expectations of patients who were going to undergo monofocal and these ones who were going to undergo multifocal lens exchange. Among the other objectives of Lowe et al. study (22) was the comparison of visual expectations of inpatients and day cases for cataract surgery.

\section{PATIENTS' SELECTION CRITERIA}

Patient selection was presented to be very crucial for the best possible assessment of patients' preoperative expectations for their visual function after a cataract surgery and most studies dealt thoroughly with patients' inclusion and exclusion criteria. The most common inclusion criterion was first-eye cataract surgery in order to avoid bias from another cataract surgery $(4,7,11-13,23)$. However, Pager et al. $(8,9)$ included also patients with a previous cataract surgery and examined how their expectations could be differentiated, compared to patients without any earlier cataract operation. Nijkamp et al. (7), who compared monofocal and multifocal IOLs, included only patients with astigmatism 1.5 diopters (D) or less, spectacle sphere power $\geq-6 \mathrm{D}$ and $\leq+4 \mathrm{D}$ and axial length between 19.5 and $26 \mathrm{~mm}$. Additionally, patients of this study should not be professional night drivers, be able to answer questionnaires in Dutch and should not have reduced mental capacity.

Among the exclusion criteria were the following: prior intraocular operation in the same eye, previous unilateral cataract surgery, single-eyed patients, corneal astigmatism, existence of psychiatric disorders and reduced mental capacity to complete the questionnaire. Patients with visually impairing ocular comorbidities such as glaucoma, macular degeneration, amblyopia, macular hole, retinal detachment or other retinal abnormalities that may affect postoperative visual performance were excluded from many studies $(2,7$, $13,22)$. Nevertheless, there is a number of studies that did not exclude patients with ocular comorbidities (10-12). Additionally, difficulty in reading or understanding the language of questionnaires was a cause of rejection $(2,7)$.

Regarding patients' age, one study conducted in Ethiopia (10) included patients aged 12 years old or older, while some other studies included patients of age $\geq 18$ years old $(11,26)$. The majority of studies included older male and female individuals, namely aged 50 years and older $(14,23)$ or $\geq 55$ years old (22).

\section{DEMOGRAPHIC CHARACTERISTICS}

In some studies, sociodemographic factors of patients including age, gender and health literacy were examined and correlated with the preoperative expectations and postoperative satisfaction. In this review, it was also examined if the level of education (low, middle or high / primary school, college, university) $(4,7,11,14,23,26,27)$ and occupation (Occupation level: I-VII) $(14,26)$ could serve as possible factors that could predict and affect patients' expectations. Finally, nervousness and eagerness for spectacle independence were investigated for their impact on the satisfaction of patients after cataract surgery (7).

\section{QUESTIONNAIRES}

Different questionnaires were used as sources of information about demographic variables and patients' preoperative expectations as regards their present difficulties in daily life and the expected improvement in visual function after the cataract operation.

Tielsch et al. (4) assessed patients' self-reported difficulty and satisfaction with their vision before and after the cataract procedure using VF-12. In addition, their preoperative expectations were measured thoroughly. Two studies $(8,9)$ which also examined the difference between expectations and final visual outcome used Visual Function Index (VF-14), which is a modification of VF-12 and one of the most common instruments used for patients who underwent cataract surgery (28). Addisu et al. (10) measured preoperative, expected postoperative and actual postoperative visual status with the use of VF-15 questionnaire (Visual Function-15, a 15-item slightly changed version of 14 -item VF-14 questionnaire). Scores on the VF-12, VF-14 and VF-15 range from 0 (no visual ability) to 100 (no visual disability) (4, 8-10). Chen et al. (11) and Mollazadegan et al. (20) used Catquest-9SF questionnaire, which contained questions about patients' troubles in everyday-life activities, their satisfaction with vision before cataract extraction and their expectations for their postoperative visual function. These expectations were compared with their self-assessed postoperative satisfaction. Nijkamp et al. (7), who conducted a study that evaluated the effectiveness of multifocal IOLs in the correction of presbyopia after cataract extraction, used Eagerness for Spectacle Independence (ESI) and the Neuroticism Scale of the Eysenck Personality Questionnaire-Revised Short Scale (EPQR-S). Other questionnaires which were used for the same purpose were the American Society of Cataract and Refractive Surgery (ASCRS) Cataract Data Collection Form (ACDCF) (16) and some self-administered questionnaires that were developed by researchers of each study $(2,12-14,21)$.

There is a considerable number of variables which were assessed in the expectation questionnaires. Among them are the ability to read small prints such as newspaper or book, read subtitles on TV, large print, numbers on mobile phone and product prices during shopping, recognize people, do fine handworks like needlework, cook, dress and have a bath by themselves, take part in the same hobbies, sports and social activities like they had before surgery and drive at daytime and nighttime $(8,10,11)$.

Regarding data collection, in order to estimate preoperative expectations and postoperative satisfaction, researchers asked patients to answer "expected questionnaires" preoperatively and other questionnaires usually 1 month $(8,9)$, 3 months $(11,12)$ or 4 months $(4,22)$ after the cataract procedure to compare postoperative outcomes and satisfaction with the expectations that they had preoperatively. Addisu et al. (10) asked patients to complete the second questionnaire 5 weeks after the surgery, while Colin et al. (2) arranged the questionnaire completion between 14 and 30 days after surgery. Patients in Nijkamp et al. study (7) filled in the postoperative questionnaires 3 months after the surgery of the first 
eye and 3 months after second-eye cataract operation. Finally, Berdeaux et al. (17) mentioned that all questionnaires in their study were filled postoperatively and recommended that it would be better if the questionnaire examining the benefits of spectacle independence was completed preoperatively in order to evaluate patients' expectations. Subsequently, questionnaire examining patient satisfaction could be answered postoperatively to evaluate their satisfaction.

\section{PATIENT PREOPERATIVE EXPECTATIONS}

In the past few decades, the revolution of cataract surgery techniques has contributed to the reduction of recovery time and the raise of expectations of patients and surgeons for postoperative visual outcome. The analysis of operation outcome could inform specialists about alterations in functional disability. For the best appreciation of the outcome of cataract surgery and, as a result, of functional ability changes, it is crucial to express this disability not only with a single variable like VA, but also in terms of visual function, quality of life and patient satisfaction (10). The desirable goal of surgeons is the best possible patients' satisfaction. However, in order to increase satisfaction, the level of preoperative expectations should be examined thoroughly.

Consequently, the examination of preoperative expectations requires the determination of some concrete terms: the definition of patients' expectations, finding ways to measure the expectations, categories of patient expectations and factors affecting patients' expectations and/or satisfaction.

\subsection{Definition of patient preoperative expectations}

According to Kravitz et al. (29) the term "patients' expectations" can be used in two different ways. "Probability expectations" are "patients' judgments about the likelihood that a set of events would occur", while "Value expectations" are defined as "patients' hopes, wishes or desires concerning clinical events".

Among the above definitions, "probability expectations" is used to describe preoperative expectations in the present review.

\subsection{Categories of patient preoperative expectations}

According to the studies of this review, the main categories of expectations measured were expectations for what they considered as "normal" or expected vision and for spectacle independence.

\subsubsection{Expectations for "normal" vision}

As regards patient expectations for normal vision, there was a variety of ways in which different researchers expressed this parameter.

Addisu et al. (10) referred that $36 \%$ of patients expected to have a postoperative VF-15 score of $100 / 100$, while Pager et al. (8) mentioned that $60 \%$ of participants had the expectation for a VF-14 score of $100 / 100$. In these two studies $(8,10)$, there is a number of patients who did not expect any improvement because they had already a preoperative VF-14 score 100 or $>90$.

$90 \%$ of individuals in Hawker et al. study (13) expected a normal vision after cataract surgery.

Nijkamp et al. (7) reported that $90.6 \%$ of patients belonging to monofocal group and $96.0 \%$ of patients belonging to multifocal group expected that their vision would be improved "much" to "very much".

According to Kara-Júnior et al. study (14), 80\% of individuals expected to find a total solution as regards the cataract.

In 1995, a study conducted by Tielsch et al. (4), demonstrated that $89 \%$ of subjects expected a postoperative VF-12 score higher than their preoperative VF-12 score. The other $11 \%$ of individuals did not expect any improvement because the majority of them had already a maximum VF-12 score of 100.

\subsubsection{Expectations for spectacle independence}

Some studies of this review examined if patients expected that they would not wear glasses after the cataract surgery and how important spectacle independence was for them.

Hawker et al. (13) observed that $73 \%$ and $87 \%$ of patients regarded spectacle independence for distant vision activities and reading respectively as an important outcome of the

Tab. 2 Comparison between preoperative expectations and postoperative perceptions of patients.

\begin{tabular}{|c|c|c|c|c|}
\hline Study & Questionnaire & $\begin{array}{l}\text { Preoperative Perceptions } \\
\text { (\%) }\end{array}$ & $\begin{array}{l}\text { Preoperative Expectations } \\
(\%)\end{array}$ & $\begin{array}{l}\text { Postoperative Perceptions } \\
\text { (Patient-reported } \\
\text { outcome) }(\%)\end{array}$ \\
\hline Chen et al. (11) & Catquest-9SF & $56.07(15.7 / 28)$ & $93.9(26.3 / 28)$ & NAt \\
\hline Addisu et al. (10) & VF-15* & 54.19 & 96.3 & 96.2 \\
\hline Pager et al. (9) & VF-14* & 84.7 & $\begin{array}{l}\text { Information video: } 83.42 \\
\text { Anatomy video: } 86.03 \\
\text { First time surgery: } 96.31 \\
\text { Previous surgery: } 95.68\end{array}$ & 89.8 \\
\hline Pager et al. (8) & VF-14* & 84.7 & 96.1 & 89.8 \\
\hline Nijkamp et al. (7) & VF-14* & $\begin{array}{l}\text { Monofocal:74.1 } \\
\text { Multifocal:71.4 }\end{array}$ & NA† & $\begin{array}{l}\text { Monofocal:96 } \\
\text { Multifocal:95.1 }\end{array}$ \\
\hline Tielsch et al. (4) & VF-12* & 80.2 & 98.7 & NAT \\
\hline
\end{tabular}

*VF = Visual Function; $† N A=$ Not applicable 
cataract surgery. Nevertheless, $73 \%$ and $85 \%$ of individuals believed that they would need distance and near glasses respectively postoperatively.

In another study (7), ESI after the lens replacement with a mono- and multifocal implant was determined. The score of ESI questionnaire, which examined perceived advantages and disadvantages of wearing glasses, ranged from 1 (very eager to be spectacle independent) to 5 (not eager at all to be spectacle independent). No significant difference between the mono- and multifocal group was found in ESI (monofocal group: $3.2 \pm 0.7$, multifocal group: $3.1 \pm 0.8, p=0.35$ ). Nijkamp et al. observed that subjects in this study believed that having good vision at more than one distance, even with complementary reading or multifocal glasses, was more important than being spectacle independent.

\section{PARAMETERS TO MEASURE EXPECTATION FULFILLMENT}

There is no doubt that there is a need for developing some novel parameters in order to quantify the outcome of cataract surgery from a different perspective. In this way, the difference between preoperative expectations and actual outcomes, in other words the expectation fulfillment, could be assessed. These parameters could either be objective or subjective. Among the objective parameters are VA and spectacle independence, while among the subjective parameters are postoperative perceptions and expectation-outcome discrepancy. Patients' preoperative perceptions regarding their visual function and preoperative expectations were compared with postoperative patient-reported outcome in Table 2.

\subsection{Visual acuity}

The measurement of VA is the most common method to estimate the postoperative outcome [Clinical Outcome Measure (COM)] (20).

Mollazadegan et al. (20) defined the improvement in VA as the increase of one line or more than one line on the Snellen chart after the surgery, while a negative COM was defined as a postoperative decline of VA.

Addisu et al. (10) described postoperative outcomes; uncorrected VA was $\geq 6 / 18$ in $57 \%$ of cases (pinhole VA $\geq 6 / 18$ : $70 \%$ ), while $29 \%$ of patients had $V A<6 / 18$. Seven patients who had $V A<6 / 60$ after 5 weeks were found to have ocular comorbidities.

Pager et al. (9) observed that there were no significant differences in postoperative VA between "expectations" and "anatomy" video group.

Pager et al. (8), in a second study, mentioned that the median VA altered from $6 / 18$ preoperatively to $6 / 6$ after the lens extraction in the operative eye.

\subsection{Spectacle independence}

Among the important parameters that could contribute to a favorable evaluation of the cataract surgery outcome is the option to offer spectacle independence.

Nijkamp et al. (7) mentioned that the monofocal group had worse Uncorrected Near Visual Acuity (UCNVA) and spectacle independence than the multifocal group. Nevertheless,
$57.3 \%$ of patients who were implanted with multifocal lenses still used spectacles for reading.

\subsection{Postoperative perceptions - Expectation fulfillment} The majority of studies described a very good proportion of patients whose expectations were met $(4,7,10,11,20)$.

Nijkamp et al. (7) observed that $62.5 \%$ from the monofocal group and $61.5 \%$ from the multifocal group achieved their expectations after the lens extraction of both eyes.

Tielsch et al. study (4) demonstrated that $61.2 \%$ of subjects had equal or better postoperative visual function compared to their preoperative expectations. However, when patients were questioned for the fulfillment of their expectations for specific daily activities, they performed better scores for each activity than for their visual function as a whole. Namely, $71 \%$ to $96 \%$ achieved their expectations of improvement for each of the VF-12 activities.

Mollazadegan et al. (20) mentioned that $10.1 \%$ of participants had a poor patient-reported outcome measure (PROM-) postoperatively, and $7.4 \%$ had a positive clinical outcome measure $(\mathrm{COM}+)$ but a negative patient-reported outcome (PROM-). They found that the latter group had difficulties in near-vision and other daily activities postoperatively. Researchers mentioned that PROM- generally was due to a good preoperative patient-reported visual function and poor VA in the best eye prior to surgery.

However, in Pager et al. study (8), where the expectations for the postoperative outcome were very high, $66 \%$ of individuals had lower perception 1 month after the cataract surgery in comparison with their preoperative expectations.

From the assessment of questionnaire answers, it was observed that there was a significant number of patients who did not achieve to meet their preoperative expectations in some daily activities, like reading small prints (eg. newspaper) $(4,8,10)$, doing fine handwork $(4,8,10)$, taking part in social activities (10), visiting friends (10) and driving at night (8).

\subsection{Expectation-outcome discrepancy}

The difference between preoperative expectations and actual outcomes is a subjective but effective way to estimate the degree of expectation fulfillment.

Chen et al. (11) noticed that the mean expectation-outcome difference was zero, namely the majority of initial expectations were met. Mainly patients implanted with aspherical $\mathrm{IOL}$ and patients with ocular comorbidity, very poor preoperative VA, poor health literacy, mild nuclear opalescence, and cortical cataract grading had a considerably greater discrepancy regarding expected and actual Catquest-9SF scores.

Pager et al. (8) observed that the mean discrepancy between expectations and actual outcomes was 6.3 points.

Examining expectation-outcome discrepancy, Tielsch et al. (4) did not find any correlation between the number of procedures performed by the surgeon per year, the period of time the ophthalmologist being in practice, the location, in which operations took place, and the discrepancy regarding expected and actual outcomes. Mean expectations of visual function were slightly but significantly higher than actual postoperative visual function for each of the provider characteristics mentioned above. 
7. DETERMINING FACTORS OF PATIENTS' EXPECTATIONS - PREDICTORS OF PATIENTS' SATISFACTION

One of the purposes of this review is the investigation of predictors of patient satisfaction by identifying the determinants of preoperative patient expectations. Patients' satisfaction is affected not only by the measured postoperative VA but also by the degree of the expectations that they have before the operation $(7,10,13,23)$. It is observed that patients with higher expectations tend to have a lower level of satisfaction compared to people who have lower expectations $(30,31)$.

\subsection{Demographic characteristics \\ 7.1.1 Age}

Hawker et al. (13) observed that, although age and expectation of needing spectacles for distance was not correlated significantly, the correlation between increasing age and expectation for near correction was weakly positive, namely older patients thought it more probable that they would need glasses for near vision than younger patients (range: between 41 to 97 years). Thus, younger patients should be treated with greater caution.

Nijkamp et al. (7) observed that older patients had a smaller ESI. This derives from the fact that 4 out of 5 patients in this study wore glasses for distance and near vision.

Tielsch et al. (4) did not find any correlation between expected postoperative scores in the VF-12 questionnaire and patients' age. However, patients $>75$ years old had a greater but not significant discrepancy between expected and postoperative scores in the VF-12 questionnaire.

\subsubsection{Gender}

A Brazilian study by Kara-Júnior et al. (14) did not observe any significant difference in expectation between the two genders as regards the possibility of full correction of cataract. Tielsch et al. (4) observed that expected postoperative VF-12 scores were not correlated with gender. Nevertheless, men seem to be satisfied more easily than women $(2,20)$.

\subsubsection{Education}

Nijkamp et al. (23) highlighted the role of patients' education in setting of realistic preoperative expectations, while Tielsch et al. (4) noticed that patient education was not correlated with expected postoperative mean VF-12 scores.

\subsubsection{Occupation-Income}

Although occupation is one of the most important affecting factors of satisfaction (6), there was not any article analyzing the relationship between occupation and preoperative expectations.

\subsubsection{Health literacy}

Chen et al. (11) concluded that low patient health literacy was associated with a significant expectation-outcome discrepancy.

\subsection{Medical information \\ 7.2.1 Patients' counseling}

According to a considerable number of studies, a discussion between the cataract surgeon and patient before the operation about the preoperative expectations for the outcome of the surgery could be very valuable. Specifically, health care professionals could inform patients about their prediction of the postoperative outcome in order to encourage more realistic expectations $(9,12,24)$.

Hawker et al. (13) emphasized the importance of discussing about the possibilities of wearing spectacles for distance and/or reading after the surgery. Thus, patients would be more likely to have realistic expectations and a high level of satisfaction.

Chen et al. (11) concluded that extensive counseling could be remarkably helpful for patients with low health literacy and systemic or ocular comorbidities because it could diminish the expectation-outcome difference and increase satisfaction.

Addisu et al. (10) resulted that patient understanding and counseling by health care professionals before surgery could help in the informing of patients about potential outcomes, decrease their expectations and, consequently, increase satisfaction even if the actual outcome is not the ideal.

Colin et al. (2) reported that $92.4 \%$ of patients included in the study said that health care professionals provided them preoperatively with enough to too much explanation regarding surgical procedure and $90.4 \%$ of patients mentioned they had received before surgery enough to too much explanation about risks of cataract surgery. Moreover, patients were questioned to answer if they asked preoperatively their surgeon all their unanswered questions and only $7.9 \%$ gave a negative answer. On the other hand, regarding the explanations they received during the surgery by their ophthalmologist, it was interesting that $67.2 \%$ of patients complained that the doctor did not give them enough explanation and only $10.3 \%$ were satisfied (received "enough" or "too much" explanation). Researchers explained that patients informed by their health care practitioners had a great level of satisfaction.

Chang-Godinich et al. (16) observed that some older patients, who had a lower level of satisfaction with their postoperative quality of life and the medical care that they received, complained of no enhancement in their functional vision in spite of the objective improvement in their VA. It was concluded that a preoperative discussion of postoperative expectations of the patients could be beneficial for them.

According to Tielsch et al. (4) patients who had been informed by ophthalmologists about the expectations that they should have during and after the surgery had a greater satisfaction. For example, a discussion of the possibility of wearing glasses for reading postoperatively or an explanation of operation risks, if patients had an ocular comorbidity, could reduce the difference between the expected and actual outcomes.

Gramer et al. (15) mentioned that patients could be informed by their physicians the day before the cataract surgery. They suggested that an informative session is less likely to address all the queries of the patient regarding his/her upcoming operation, and a more integrated approach including written material should be preferred. 


\subsubsection{Video presentation}

In their study, Pager et al. (9) showed patients who were scheduled for cataract operation one of two different videos. The first videotape informed patients about the procedure of cataract surgery and its possible dangers ("expectations video group"), while the second video described only the way of cataract formation ("anatomy video group"). It is worth noting that although the expectations video group in comparison with the expectations of the second group feared preoperatively that the surgery would have more risks and discomfort, patients who watched the first informational videotape, had a better understanding of the procedure of the extraction of their lens, less anxiety and a greater satisfaction, probably because they felt better that the procedure was not as painful and dangerous as they expected.

\subsubsection{Comorbidity}

The discrepancy regarding expected and actual outcomes after a cataract procedure is undoubtedly correlated with ocular and systemic comorbidities (11), which are the most important factors that can predict patient dissatisfaction (8, 16). For this reason, many surgeons hesitate to undertake the operation of patients with ocular or systemic comorbidities that may affect their visual capacity. Therefore, physicians try to explain the risks, give an estimate of the postoperative visual capacity and, overall attempt to manage preoperative expectations, accordingly (12).

Chen et al. (11) came to the conclusion that patients with systemic and ocular comorbidity should have an educative discussion with their ophthalmologist in order to reduce the expectation-outcome discrepancy.

Kuo et al. (12) mentioned that all patients with ocular or systemic comorbidity, including keratectasia after Laser In Situ Keratomileusis (LASIK), Age-Related Macular Degeneration (ARMD), cerebrovascular accident and retinitis pigmentosa, had a low possibility to meet their preoperative expectations of visual function after the cataract surgery.

Addisu et al. (10) observed that the existence of ocular comorbidities was one of the most important predictive factors of dissatisfaction. On the contrary, they suggested poor correlation between patients' satisfaction and enhancement of visual function.

\subsubsection{Unrealistic Expectations}

Sometimes patients have an unreasonably high level of expectations. The most common expectations are reading small prints, doing fine handwork and driving at night $(8,10)$. In this case, it is very common that the majority of this patient group cannot meet their preoperative expectations $(4,8)$.

Tielsch et al. (4) were surprised by the fact that participants with ocular comorbidities or with generally low prognosis had the same expectations in VF-12 scores as patients with favorable predictive factors. However, there was a significant difference between the actual and the expected outcomes. As a result, they had a greater expectation-outcome discrepancy compared with younger patients without comorbidity.

Pager et al. (8) emphasized that there was a number of patients who had no realistic expectations such as driving at night, reading small prints and doing fine handwork. In addition, it was demonstrated that the control of patient expectations is more important than the actual improvement of postoperative outcomes for the best possible satisfaction.

Chang-Godinich et al. (16) reported that the fact that patients did not perceive a better postoperative visual function probably derived from the absence of realistic expectations.

\subsubsection{Spectacles preoperatively}

One of the major patients' expectations is postoperative spectacle independence (32). According to Hawker et al. (13), who recommended the consideration of the patients' preoperative refractive status, patients who needed glasses before the cataract surgery generally expected to use them also after surgery. On the other hand, a small number of patients who did not wear spectacles preoperatively, had the expectation of complete spectacle independence. However, these high expectations increased the risk for dissatisfaction.

Nijkamp et al. (7) observed that older patients, who preoperatively in their great majority needed spectacles both for distance and near vision, did not have very high expectations and were satisfied even if they should use glasses after the surgery, too. In addition, in this study, where the efficacy of multifocal intraocular lenses (IOLs) to correct presbyopia at the same procedure with cataract correction was examined, it was highlighted that patients having ESI would accept seeing halos and having low contrast sensitivity, which are some side effects of multifocal IOLs, as long as they would not use spectacles again $(7,25)$.

\subsubsection{Cost of surgery}

Wei et al. (18) described a cataract surgery payment model and assessed the satisfaction of patients according to the type of payment. The first type ["National Health Insurance (NHI) coverage"] was the total cost coverage of a "general IOL" by the NHI, while the second type ("balance billing") was the partial payment depending on the kind and price of IOL (monofocal/multifocal, yellow-tinted or not). It is worth to mention that there was not difference in satisfaction between the two groups. Writers supposed that this was due to the lower degree of expectations of patients using $\mathrm{NHI}$ coverage and, as a result, due to the low expectation-outcome discrepancy. On the contrary, patients who chose to pay for their procedure, expected to receive the best possible medical services. For this reason, they had a higher level of expectations and a lower level of satisfaction, although there was not any difference in VA between the two groups. Actually, patients who were implanted with multifocal and yellow-tinted IOLs) had an easier looking at small objects and objects under strong lighting conditions.

\subsubsection{Personality-Psychology}

In this review, studies did not reveal a concrete relationship between personality or emotional status and patients' expectations. Patient's personality and emotions were connected only with satisfaction.

Nijkamp et al. (7) examined the influence of patient personality on their satisfaction and the possibility of taking the 
personality of patients into account in order to predict their satisfaction. It was hypothesized that it was more difficult for patients with obsessive personalities (eg. neuroticism) to be satisfied. Nevertheless, according to the results of this study, neuroticism did not seem to be related with satisfaction. Consequently, obsessiveness was found to be an inappropriate criterion for the inclusion of patients for cataract operation with multifocal IOLs.

Another study, which reveals how difficult the prediction of visual satisfaction is, is the study of Prakash et al. (19), who explained that expectations and mindset of patients can bias questionnaire outcomes because each patient perceives symptoms in a different personal way.

The study of Yucelt et al. (6) demonstrated that patients who were not satisfied with their life quality and are emotionally unstable (eg. having depression or other health problems) are more likely to be also dissatisfied with the received health care. For this reason, medical care professionals should always take patient expectations and emotions into consideration.

\subsubsection{Previous cataract surgery}

The findings in Pager et al. (9) study revealed that there was a difference in the preoperative expectations and perceived postoperative outcomes between patients with and without a previous cataract surgery. Analytically, patients who had experienced a previous cataract operation expected that they would be less anxious and the procedure would be more comfortable in comparison with patients who did not have such a previous experience. Postoperatively, patients who had previously experienced cataract surgery were more likely to find the surgery close to their expectations.

\section{ADVANTAGES OF PATIENTS' SATISFACTION}

Beyond the improvement of patients' vision and life quality, the effort to raise their satisfaction has some additional advantages for health care providers. Patients whose preoperative expectations have been met, usually have a better compliance with medication, a consistent follow-up and generally enjoy the benefits of the procedure to the maximum degree (8, 9). Furthermore, satisfied patients do not lose their relationship with their physicians, recommend them to other people and do not blame them judicially for malpractice $(8-10,26)$.

\section{DISCUSSION}

Nowadays, higher life expectancy is likely to increase the number of patients having cataract, which is a significant cause of low vision not only in developing but also in developed countries (1). For this reason, further knowledge of the factors that could affect the outcome of a cataract surgery would aid in the improvement of treatment quality.

Patient satisfaction depends both on the actual outcomes of VA and on the preoperative expectations of patients. Satisfaction and visual outcomes have a proportional relationship, while satisfaction is inversely proportional to preoperative expectations. The objective of health care systems is to offer high quality services. Therefore, addressing this objective is possible either by the improvement of the actual visual outcomes that patients attain or by the reduction of their preoperative expectations. However, cataract-related technological advances, cannot cope with the continuously increasing demand for perfect vision. Consequently, medical care professionals should now focus on the influence of preoperative expectations, too. Namely, ophthalmologists should try to reduce their patients' expectations to a realistic level that is compatible to their setting and expertize (12).

As regards the assessment of patients' expectations before cataract surgery, the majority of relevant studies used predefined psychometric tools and correlated their results with a series of visual capacity parameters.

Moreover, individuals were asked about their preoperative expectations both for expected visual capacity and for spectacle independence. Despite the fact that accurate assessment of both aforementioned expectations is essential to the care providers, no common methods of estimating them have been introduced. For instance, some studies measured the mean expected postoperative questionnaire score; the maximum score of Catquest-9SF is 28 (11), while the maximum VF score is 100 . Another study (8) measured the percentage of subjects that had a maximum expectation score. Other studies expressed the portion of participants who expected a normal vision (13), "much" to "very much" improvement (7) or a full recovery from the cataract (14). In an additional study (4), researchers assessed the number of patients who expected better postoperative visual function scores in comparison to the preoperative ones. Regarding the estimation of expectations for spectacle independence, there was one study (13) that examined this parameter too. A study examining ESI (7) found that individuals thought that having good vision at more than one distance, even with complementary reading or multifocal glasses, was more significant than not wearing glasses. However, the reliability of these results should be confirmed with further investigation. Therefore, it would be valuable if expectations about spectacle independence were examined on a regular basis. Consequently, it is obvious that there is no standard way of measurement and evaluation of patient expectations. As a result, the difficulty in comparison of patients' expectations between the studies of this review of literature was considerable.

Postoperative VA and spectacle independence are useful for the comparison with the preoperative status. However, they are not enough for a total evaluation of the success of a cataract procedure. This review indicated that patients' perception of their visual function should be asked after the surgery $(4,7,8,10,11,20)$. This would be necessary in order for ophthalmologists to know if patients' expectations were fulfilled. The majority of studies $(4,7,8,10,11,20)$ mentioned the percentage of patients who addressed or surpassed their preoperative expectations for the minimal-required visual capacity to complete their daily activities. However, only a small number of studies described patient-reported outcomes for each daily activity $(4,8,10)$. Within this context, the most difficult daily activities that usually were not addressed following cataract-extraction surgery were revealed. Among them, reading small prints and doing fine handwork $(4,8,10)$. Moreover, patients who had been implanted with multifocal lenses had higher expectations for specific activities; among them driving at night (8). Undoubtedly, it would be very useful if all studies examining patient expectations 
for cataract removal in the future evaluated expectations and postoperative perceptions for each activity separately with a special questionnaire. In this way, researchers could detect weaknesses of each type of lenses and try to improve them for the achievement of the best possible result.

We assumed that a possible reason that some studies showed a lower postoperative perception than preoperative expectations was ECCE instead of phacoemulsification. ECCE was the treatment for cataract in a study of the previous decade (9) and in a study about cataract surgery in a developing country (10), where the surgical techniques and the equipment were not very advanced. However, the number of studies using ECCE and the total number of studies examining the postoperative perceptions was limited.

Concerning the time when follow-up and questionnaire completion about patients' postoperative expectation fulfillment took place, this review indicated that there was no common method. Some of the studies collected data 1 month $(8,9)$ after cataract operation, other studies collected them 3 months $(11,12)$ and other studies 4 months $(4,22)$ postoperatively. Consequently, it is possible that this difference could complicate the objective comparison of postoperative perceptions because visual function is gradually improved over time postoperatively. Further investigation is required in order to clarify the most appropriate period of time after lens exchange for the most reliable assessment of vision improvement compared with patients' expectations.

A final parameter which was taken into account in a significant number of studies $(4,7,8)$ was the expectation-outcome discrepancy. Specifically, the difference between expected and actual outcome was estimated. This parameter should constitute a standard examination object of the studies concerning patient expectations as regards cataract surgery because it is a simple way to evaluate the success of a cataract operation.

In summary, our review suggests that further investigation is required in order to create a protocol that should be followed in every study concerning patients' preoperative expectations. Namely, VA before and after cataract surgery should be measured. Preoperative expectations both for spectacle independence and normal vision at more than one distance, even with complementary reading or multifocal glasses, should be estimated prior to cataract surgery. Furthermore, an indispensable part of the postoperative approach of patients should be the assessment of their postoperative spectacle independence, patients' perception of their visual function with the completion of a respective questionnaire and the expectation-outcome discrepancy.

There are not many studies examining patients' preoperative expectations for the outcomes of a cataract surgery and ophthalmological operations in general, while there is a plethora of studies examining patients' satisfaction as regards lens extraction. Additionally, nowadays, an increasing number of patients are implanted with multifocal IOLs. As a result, they have high expectations for their vision at all distances (distance, intermediate, near vision) and under all lighting conditions. However, there is an even greater deficiency in studies examining patients' preoperative expectations for cataract surgery with implantation of a multifocal lens. For this reason, further studies are needed in order to evaluate the relationship between preoperative expectations and postoperative outcomes of patients implanted not only with monofocal but also with multifocal IOLs.

With regard to the factors that are able to influence patient expectations, this systematical review of literature indicates that there is a variety of determinants of preoperative expectations. Ocular and systemic comorbidity, unrealistic expectations, preoperative spectacle independence, the cost of surgery, a previous cataract surgery and the level of health literacy are the major factors that can affect positively or negatively patient preoperative expectations. On the other hand, although it is known that demographic characteristics such as age, gender, education, occupation and income are associated with satisfaction $(2,26,27)$, to our knowledge, there are not many studies which examine the correlation between age/ gender/education and expectations, and there is no study regarding the occupation/income-expectations relationship.

It was hypothesized that patients' personality and psychological state could play a determinant role in the development of patients' initial expectations. Nevertheless, all studies that were found correlated emotional state only with satisfaction. Obsessiveness, neuroticism and depression could be factors that affect negatively patient satisfaction. Regarding preoperative expectations, we assume that patients that are not emotionally stable have unpredictable preoperative expectations. Further studies are needed to clarify the relationship between emotional instability and patients expectations.

\section{CONCLUSIONS}

Our intention was the best possible comprehension of the literature regarding patient expectations for a favorable cataract surgery visual outcome and the assistance of other researchers in further investigation in this domain of knowledge. Evaluation of patients' preoperative expectations provides essential information to the ophthalmologist. This review suggests that the final postoperative perception could be affected by the actual outcome of the operation and by patient preoperative expectations. The higher the actual visual outcome and the lower the preoperative expectations are, the better the postoperative perception of visual outcome is. In addition, our review emphasizes the importance of the assessment of factors that determine patients' preoperative expectations. Demographic characteristics such as age and the level of health literacy, as well as other factors including ocular and systemic comorbidity, unrealistic expectations, preoperative spectacle independence, the cost of surgery, and a previous cataract surgery could affect preoperative expectations and predict patients' satisfaction. Taking these factors into consideration could lead to more accurate prediction of patient satisfaction and better control of expectations with an extensive preoperative counseling.

\section{FINANCIAL DISCLOSURE}

No financial support was received for this study. None of the authors has any proprietary interests or conflicts of interest related to this submission. It is not simultaneously being considered for publication at any other journal. 


\section{REFERENCES}

1. WHO. Cataract, Priority eye diseases, Prevention of Blindness and Visual Impairment. (Accessed July 2, 2018 at http://www.who.int/blindness /causes/priority/en/index1.html).

2. Colin J, El Kebir S, Eydoux E, Hoang-Xuan T, Rozot P, Weiser M. Assessment of patient satisfaction with outcomes of and ophthalmic care for cataract surgery. J Cataract Refract Surg 2010; 36(8): 1373-9.

3. Apple DJ, Peng Q, Visessook N, et al. Surgical prevention of posterior capsule opacification. Part 1: Progress in eliminating this complication of cataract surgery. J Cataract Refract Surg 2000; 26: 180-7.

4. Tielsch JM, Steinberg EP, Cassard SD, et al. Preoperative functional expectations and postoperative outcomes among patients undergoing first eye cataract surgery. Arch Ophthalmol 1995; 113(10): 1312-8.

5. McAlinden C. The importance of doctor-patient communication. Br J Hosp Med (Lond) 2014; 75(2): 64-5.

6. Yucelt U. An investigation of causes of patient satisfaction/dissatisfaction with physician services. Health Mark Q 1994; 12(2): 11-28.

7. Nijkamp MD, Dolders MG, de Brabander J, van den Borne B, Hendrikse F, Nuijts RM. Effectiveness of multifocal intraocular lenses to correct presbyopia after cataract surgery: a randomized controlled trial. Ophthalmology 2004; 111(10): 1832-9.

8. Pager CK. Expectations and outcomes in cataract surgery: a prospective test of 2 models of satisfaction. Arch Ophthalmol 2004; 122(12): 1788-92.

9. Pager CK. Randomised controlled trial of preoperative information to improve satisfaction with cataract surgery. Br J Ophthalmol 2005; 89(1): 10-3.

10. Addisu Z, Solomon B. Patients' preoperative expectation and outcome of cataract surgery at jimma university specialized hospital-department of ophthalmology. Ethiop J Health Sci 2011; 21(1): 47-55.

11. Chen Z, Lin X, Qu B, et al. Preoperative Expectations and Postoperative Outcomes of Visual Functioning among Cataract Patients in Urban Southern China. PLoS One 2017; 12(1): e0169844.

12. Kuo IC, Broman AT, Massof RW, Park W. The impact of cataract surgery on patients from a low-vision clinic. Can J Ophthalmol 2011; 46(5): 391-8.

13. Hawker MJ, Madge SN, Baddeley PA, Perry SR. Refractive expectations of patients having cataract surgery. J Cataract Refract Surg 2005; 31(10): 1970-5.

14. Kara-Júnior N, Temporini ER, Kara-José N. Cataract surgery: expectations of patients assisted during a community project in São Paulo, state of São Paulo, Brazil. Rev Hosp Clin Fac Med Sao Paulo 2001; 56(6): 163-8.

15. Gramer E, Leydhecker W, Krieglstein GK. The physician's obligation to educate patients - legal aspects-patients' expectations. Klin Monbl Augenheilkd 1982; 181(1): 46-53.

16. Chang-Godinich A, Ou RJ, Koch DD. Functional improvement after phacoemulsification cataract surgery. J Cataract Refract Surg 1999; 25(9): 1226-31.
17. Berdeaux G, Meunier J, Arnould B, Viala-Danten M. Measuring benefits and patients' satisfaction when glasses are not needed after cataract and presbyopia surgery: scoring an hometric validation of the Freedom from Glasses Value Scale (FGVS). BMC Ophthalmol 2010; 10: 15

18. Wei CK, Wang SM, Lin JC. A study of patient satisfaction after cataract surgery with implantation of different types of intraocular lenses. BMC Res Notes 2012; 5: 592.

19. Prakash G, Prakash DR, Agarwal A, Kumar DA, Agarwal A, Jacob S. Predictive factor and kappa angle analysis for visual satisfactions in patients with multifocal IOL implantation. Eye (Lond) 2011; 25(9): 1187-93.

20. Mollazadegan K, Lundström M. A study of the correlation between patient-reported outcomes and clinical outcomes after cataract surgery in ophthalmic clinics. Acta Ophthalmol 2015; 93(3): 293-8.

21. Silveira JA, Hayashi L, Scarpi MJ. Identification of patients' needs and expectations in a cataract clinic connected with a university public hospital. Arq Bras Oftalmol 2005; 68(5): 639-44.

22. Lowe KJ, Gregory DA, Jeffery RI, Easty DL. Patient perceptions and social impact. Preliminary results of the Bristol MRC Study. Eye (Lond) 1991; 5(Pt 3): 373-8.

23. Nijkamp MD, Nuijts RM, Borne B, Webers $C A$, van der Horst F, Hendrikse F. Determinants of patient satisfaction after cataract surgery in 3 settings. J Cataract Refract Surg 2000; 26(9): 1379-88.

24. Zuo L, Zou H, Fei X, Xu W, Zhang J. The impact of unilateral or bilateral cataract surgery on visual acuity and life quality of elderly patients. J Ophthalmol 2015; 2015: 509049.

25. Dick HB, Krummenauer F, Schwenn O, Krist R, Pfeiffer N. Objective and subjective evaluation of photic phenomena after monofocal and multifocal intraocular lens implantation. Ophthalmology 1999; 106(10): 1878-86.

26. Yucelt U. An investigation of causes of patient satisfaction/dissatisfaction with physician services. Health Mark Q 1994; 12(2): 11-28.

27. Baron-Epel O, Dushenat M, Friedman N. Evaluation of the consumer model: relationship between patients' expectations, perceptions and satisfaction with care. Int J Qual Health Care 2001; 13(4): 317-23.

28. Labiris G, Giarmoukakis A, Patsiamanidi M, Papadopoulos Z, Kozobolis VP. Mini-monovision versus multifocal intraocular lens implantation. J Cataract Refract Surg 2015; 41(1): 53-7.

29. Kravitz RL. Patients' expectations for medical care: an expanded formulation based on review of the literature. Med Care Res Rev 1996; 53(1): $3-27$.

30. Berdeaux G, Viala M, Roborel de Climens A, Arnould B. Patient-reported benefit of ReSTOR multi-focal intraocular lenses after cataract surgery: results of principal component analysis on clinical trial data. Health Qual Life Outcomes 2008; 6: 10.

31. Unsal U, Baser G. Evaluation of Different Power of Near Addition in Two Different Multifocal Intraocular Lenses. J Ophthalmol 2016; 2016: 1395302.

32. Pager CK, McCluskey PJ, Retsas C. Cataract surgery in Australia: a profile of patient-centred outcomes. Clin Exp Ophthalmol 2004; 32(4): 388-92. 